$\mathrm{mm}$ under direct visualization using monopolar cautery. The mucosa edges were reapproximated with V-lock suture.

Results Pathology showed squamous mucosa with high-grade vaginal intraepithelial neoplasia and a focal area of invasion (with depth of invasion $<1 \mathrm{~mm}$ ). The patient tolerated the procedure well and was discharged from Day Surgery without any postoperative complications.

Conslusion Transvaginal endoscopic resection for superficially invasive vaginal lesions can be performed safely and provide accurate diagnosis with excellent visualization.

\section{SF012/\#204 10 STEPS TO APPROACH LARGE OVARIAN MASSES THROUGH VNOTES (VAGINAL NATURAL TRANSLUMINAL ENDOSCOPIC SURGERY)}

'L Badiglian-Filho*, ${ }^{1} \mathrm{G}$ Baiocchi, ${ }^{1} \mathrm{C}$ Chaves Faloppa, ${ }^{2}$ J Baekelandt. ${ }^{1}$ AC Camargo Cancer Center, Gynecologic Oncology, Sao Paulo, Brazil; '2Imelda Hospital, Gynecologic Oncology, Bonheiden, Belgium

\subsection{6/ijgc-2021-IGCS.56}

10 steps to approach large ovarian masses through vNOTES. Introduction VNOTES (vaginal Natural Orifices Transluminal Endoscopic Surgery) is a novel technique that allows a laparoscopic approach to the pelvic and abdominal cavity through the vagina. It seems to facilitate large ovarian masses (with benign characteristics) removal when compared to conventional laparoscopy.

Description We recommend 10 steps to approach large ovarian masses through vNOTES. Each step is explained in the surgical video.

Step 1. Select suitable case.

Step 2. Setting up the patient and the surgical team.

Step 3. Accessing the cavity.

Step 4. Inserting vaginal port.

Step 5. Cavity inspection.

Step 6. Sealing and division.

Step 7. Bag the cyst.

Step 8. Cavity revision.

Step 9. Revise the inner ring of the vaginal port.

Step 10. Closing the vaginal vault.

Conclusion It is feasible to approach large ovarian masses through vNOTES.

\section{SF013/\#236 ROBOTIC APPROACH FOR A TOTAL HYSTERECTOMY BILATERAL SALPINGO- OOPHORECTOMY AND SUCTION CURETTAGE OF A 20-WEEK SIZE UTERUS WITH GESTATIONAL TROPHOBLASTIC NEOPLASIA}

${ }^{1} V$ Gupta*, ${ }^{2} S$ Salvador, ${ }^{2}$ W Gotlieb, ${ }^{2} S$ Lau. 'McGill University, Gynaecologic Oncology, Montreal, Canada; ${ }^{2}$ McGill University, Jewish General Hospital, Gynecology Oncology, Montreal, Canada

\subsection{6/ijgc-2021-IGCS.57}

Introduction Gestational trophoblastic neoplasia (GTN) is a malignant trophoblastic disease following either molar or nonmolar pregnancies. GTN is primarily treated through uterine evacuation using suction curettage followed by observation or adjuvant chemotherapy based on WHO risk scoring. ${ }^{1,2}$ In patients who have completed child-bearing, hysterectomy is an acceptable option and may decrease time to remission and required chemotherapy cycles. ${ }^{2}$ In patients presenting with large volume uterine disease, evidence of metastasis, and highrisk WHO scoring, patients are treated with multi-agent chemotherapy including Etopisode, Methotrexate, Actinomycin-D, Cycophosphamide, and Vincristine (EMA-CA). ${ }^{1,2}$ EMA-CO has significant risks of complications, including acute hemorrhage and trophoblastic emboli. ${ }^{2,3}$ In patients with large uteri, surgical risks include uterine perforation and acute hemorrhage, ${ }^{4}$ requiring a large laparotomic incision. Accordingly, there is a need for risk-reducing minimally invasive approaches in the surgical treatment of GTN.

Description The patient is a 53 year old G4P4 presenting with an enlarged uterus of $20 \mathrm{~cm}$ with snowstorm appearance, a beta-hCG >400 000IU, lung metastases and a WHO risk score of 8 . Preoperatively her blood pressure was 168/105. She underwent a robotic total hysterectomy and bilateral salpingo-oophorectomy, and guided suction curettage. Blood loss was minimal. The patient was scored post-procedure as WHO low risk (3). She received methotrexate, and switched to Actinomycin-D after a plateau in beta-hCG. Her beta-hCG is normal 5 months later.

Conclusion We present a minimally invasive approach that ameliorates the surgical and chemotherapy risks of uterine rupture, acute hemorrhage, and trophoblastic emboli, with a normalization of beta-hCGs after treatment with single-agent chemotherapy.

\section{SF014/\#370 LAPAROSCOPIC REPAIR OF OBTURATOR NERVE INJURY AT THE TIME OF PELVIC LYMPHADENECTOMY}

R Linder*, J Gomez, T Feigenberg. Trillium Health Partners, Credit Valley Hospital, Gynecology Oncology, Mississauga, Canada

\subsection{6/ijgc-2021-IGCS.58}

Introduction The obturator nerve is formed by the lumbar plexus, receiving its fibers from the anterior division of L24. Clinically, obturator nerve injury manifests with sensorial loss at the medial aspect of thigh, pain at medial portion of the groin and ipsilateral adductor weakness. The risk of obturator nerve injury is increased during pelvic lymphadenectomy and surgeries for gynecologic malignancies. Here, we present a video film of full-thickness transection of obturator nerve at the time of pelvic lymphadenectomy in a patient who underwent laparoscopic surgery for endometrial cancer. The transected nerve was primarily repaired through a laparoscopic approach at the time of surgery. The patient experienced a full recovery with no motor function deficiencies.

Description In this surgical film we present an 83-year-old woman, G2P2, who was diagnosed with a grade 3 endometrioid endometrial adenocarcinoma. She underwent a laparoscopic hysterectomy and sentinel lymph-node biopsies as well as resection of enlarged external iliac node. During the procedure, we identified a complete transection of the obturator nerve. To enable an end to end repair, the external iliac artery and vein were mobilized. The proximal end of the nerve was identified lateral to these vessels. An end-to-end anastomosis of the nerve was performed using Prolene sutures. The patient experienced an uneventful post-operative recovery. She discharge home on post-operative day 2 , able to ambulate independently with no assistance. 
Conclusion Obturator nerve injuries are a well-recognized complication of pelvic lymphadenectomy. Immediate laparoscopic nerve repair, can facilitate earlier motor recovery and prevent the need for laparotomy.

\section{SF015/\#465 PELVIC LYMPHADENECTOMY MADE EASY - A STEPWISE TECHNIQUE FOR SYSTEMATIC PELVIC LYMPH NODE DISSECTION}

S Smyth*, C Pappa, H Jiang, M Alazzam. Oxford University Hospitals NHS Foundation Trust, Gynaecological Oncology, Oxford, UK

10.1136/ijgc-2021-IGCS.59

Introduction Systematic pelvic lymph node dissection is a standard procedure conducted in the management of cervical and high-risk endometrial cancers. This requires a precise understanding of anatomical landmarks in addition to surgical approach, with present's confusion for trainees amidst multiple learning techniques. Trainees must develop economy of movement and maximise lymph node yield whilst avoiding potential complications including injury to nerves and vessels as well as post-operative morbidity.

Description Identify the boundaries - Lateral border - develop retroperitoneal space by division of round ligament laterally, divide peritoneum along the medial border of psoas muscle with identification of genitofemoral nerve toward paracolic gutter - Medial border - obliterated umbilical artery - dissect along lateral border to level of common iliac vessels bifurcation - Inferior border - obturator nerve - identify obturator foramen following pubic bone with nerve seen below - Caudal border - deep circumflex vessels - Cranial border - bifurcation of common iliac vessels - Lymph node en-bloc dissection technique - Orientation from lateral to medial and caudal to cranial - Initiate from lateral and caudal borders and dissect from external iliac vessels - Once below external iliac vein follow pubic bone to identify obturator nerve as it crosses the obturator foramen, acknowledging corona mortis vessel - Collect lymph nodes within anatomical boundaries - Ensure haemostasis.

Conclusion/Implications We present this case as an aide memoire of a basic gynaecological oncology technique. This is an essential surgical skill to develop with knowledge and practice for all subspecialists in training. https://www.dropbox.com/s/ b2k20eph9aomb5a/BPLND\%20IGCS.mp4?dl=0

\section{SF016/\#501 TECHNIQUE OF ROBOTIC ASSISTED RADICAL HYSTERECTOMY WITH BILATERAL PELVIC LYMPH NODE DISSECTION FOR EARLY CERVICAL CANCER}

J Thingujam*, R Ranade, S Dhevi RS. Narayana Mazumdar Shaw Medical Centre, Gynecological Oncology, Bangalore, India

\subsection{6/ijgc-2021-IGCS.60}

Introduction Cervical cancer is the $2^{\text {nd }}$ most common cancer in Indian women (as per globocan 2018). For many years, radical hysterectomy is the treatment of choice for early stage cervical cancer. This procedure has been traditionally performed via laparotomy but with the introduction of robotic assisted radical hysterectomy, blood loss during procedure, hospital stay, post-operative complications is relatively reduced.
Description The video illustrates a sequential narrative of operative steps of our robotic assisted radical hysterectomy and bilateral pelvic lymph node dissections in a 46-year-old female with squamous cell carcinoma of cervix. She presented with proliferative growth of $2 \times 2 \mathrm{~cm}$ in the cervix, fornices free, bilateral parametrium and rectal mucosa free. The urinary bladder was dissected downward, the retroperitoneal space opened and visualized important structures like ureters and iliac vessels. Uterine arteries cauterized, clipped and cut at the level of its origin, ureters dissected from medial leaf of broad ligament peritoneum down to its entrance into the parametrium tunnel of Wertheim. Pararectal and paravesical space created. The infundibulopelvic ligament cauterized and cut laterally, pelvic wall peritoneum of broad ligament incised downward to base of uterosacral ligament, uterosacral ligament cauterized and cut closed to rectum. Colpotomy done specimen delivered vaginally without spillage. Bilateral pelvic lymph node dissection was done and specimen delivered vaginally. vault closure done with v- lock sutures.

Conclusion Certain studies demonstrate the safety and feasibility of Robotic assisted radical hysterectomy for early-stage cervical cancer. It provides benefits such as less bleeding, reduced in hospital stay and decrease in post-operative complication

\section{SF017/\#513 PRESERVATION OF LEFT TRIPLE RENAL ARTERY DURING LAPAROSCOPIC PARA-AORTIC LYMPHADENECTOMY}

JM Eom, JS Choi*, YK Kim. Hanyang College of Medicine, Obstetrics and Gynecology, Wangsimni-ro, Seongdong-gu, Seoul, Korea, Republic of

\subsection{6/ijgc-2021-IGCS.61}

Objective To demonstrate of preservation of left triple renal artery during laparoscopic para-aortic lymphadenectomy.

Design Case study.

Settings University hospital in Korea. Patients: A 54-year-old Korean woman with postmenopausal bleeding and thickened endometrium $>3 \mathrm{~cm}$ presented to our department. The histopathology of biopsied endometrium revealed grade 2 endometrioid adenocarcinoma. The preoperative MRI shows an about $6 \mathrm{~cm}$ sized large volume of tumor within the endometrial cavity.

Interventions We perform the laparoscopic staging surgery. Firstly we performed peritoneal washing cytology, LAVH, BSO, pelvic lymphadenectomy. We designated four area for para-aortic lymphadenectomy. During the procedure in area 4, it was confirmed that two left renal arteries were derived from the trunk of the aorta below the left renal vein. The left lower segmental artery was derived from the middle part of the inferior mesenteric artery and left renal vein. The middle segmental artery was derived just below left renal vein. The left main renal artery was located on the dorsal side of the left renal vein at its normal position. We carefully resected the para-aortic lymph nodes to prevent variant renal artery damage.

Conclusions Laparoscopy is a feasible and safe approach to diagnosis of vascular anomaly during para-aortic lymphadenectomy for gynecologic malignancies. It is very important for the gynecologic oncologist to have knowledge of retroperitoneal vascular anatomy, experience in laparoscopic surgery, and an accurate surgical technique to avoid vascular injury during laparoscopic para-aortic lymphadenectomy. 